CASE REPORT

\author{
T. Iwata \\ T. Mori \\ H. Tajiri
}

\section{Successful Staged Endovascular Treatment of a Symptomatic Cervical Carotid Bifurcation Stenosis Coupled with a Coincidental Unruptured Cerebral Aneurysm in the Carotid Distal Segment}

SUMMARY: A 69-year-old woman had a symptomatic cervical carotid stenosis coupled with a coincidental unruptured cerebral aneurysm in the carotid distal segment. She underwent endovascular coiling for the aneurysm and then carotid stent placement (CAS) 1 month later. Both lesions were treated successfully and neither complications nor strokes occurred after the procedures. Staged endovascular treatment of coiling and subsequent CAS may be safe for patients with a cervical carotid stenosis coupled with cerebral aneurysms.
$\mathbf{P}$ atients with a symptomatic cervical carotid bifurcation stenosis coupled with a coexisting cerebral aneurysm in the carotid distal segment present a therapeutic dilemma. ${ }^{1}$ Relief of the carotid stenosis, leading to a sudden rise in perfusion pressure, might increase the potential risk of aneurysm rupture. ${ }^{1-4}$ Conversely, repair of aneurysms with the patients under general anesthesia may increase the risk of strokes because of decreased cerebral blood flow (CBF) imposed by the carotid stenosis. ${ }^{1,2}$ Although endovascular therapy may be essential for patients with a symptomatic carotid stenosis coupled with an unruptured cerebral aneurysm in the distal segment, a therapeutic strategy has not yet been established. If a carotid stenosis is dilated by stent placement first, a sudden rise in perfusion pressure and subsequently required high-dose antiplatelets may increase the risk of aneurysm rupture. Even though aneurysm coiling first is a rational approach, there are technical problems concerning how to embolize aneurysms through the cervical carotid bifurcation stenosis.

We hereby report a case of successful staged endovascular treatment of aneurysm coiling and subsequent carotid stent placement (CAS).

\section{Case Report}

A 69-year-old woman experienced a transient episode manifesting as a right-sided hemiparesis. She had a history of hypertension and diabetes mellitus for at least 10 years, which had been under fair control with multiple medications. She also had unstable angina and was treated by percutaneous coronary intervention. Her father died of subarachnoid hemorrhage. Findings of a neurologic examination on admission were normal. MR imaging showed a mild ischemic change in the territory of the left middle cerebral artery, and MR angiography showed a high-grade stenosis of the left internal carotid artery (ICA) and an incidental intracranial aneurysm of the ipsilateral ICA. Left carotid angiography revealed an 80\% stenosis of the left ICA (Fig 1A) and a coincidental unruptured intracranial aneurysm $(6 \times 4 \mathrm{~mm})$ in the distal segment (Fig 1B).

With the patient's fully informed consent, staged endovascular

Received March 10, 2008; accepted after revision April 16.

From Shonan Kamakura General Hospital, Kamakura, Japan.

Please address correspondence to Takahsia Mori, MD, PhD, Stroke Treatment, Shonan Kamakura General Hospital, 1202-I Yamazaki, Kamakura, 247-8533, Japan; e-mail: morit-koc@umin.net

DOI 10.3174/ajnr.A1172 procedures were attempted. On June 27, 2007, coiling of the aneurysm was performed first under local anesthesia. A guiding catheter (6F Shuttle-SL guide sheath with 0.087 internal diameter; Cook, Bloomington, Ind) was positioned in the left common carotid artery proximal to the left carotid bifurcation stenosis. For the stability of the guide catheter during the procedures, a guidewire $(0.014 \mathrm{inch}, 300 \mathrm{~cm}$ in length, Right Away Super Hard; Piolax, Kanagawa, Japan) was inserted into the external carotid artery (ECA) (Fig 2A). Then, a microcatheter (Excelsior SL-10; Boston Scientific, Fremont, Calif) was placed through the carotid bifurcation stenosis, and there was still adequate flow into the ipsilateral cerebral hemisphere. No ischemic symptoms occurred after insertion of the microcatheter into the carotid bifurcation stenosis and during the procedures. The microcatheter was navigated into the cerebral aneurysm through the carotid bifurcation stenosis, and aneurysm coiling was performed with Guglielmi detachable coils (Boston Scientific) and Trufill coils (Cordis, Miami Lakes, Fla) and was accomplished successfully. She received 2 antiplatelet agents (aspirin, $100 \mathrm{mg} /$ day, and clopidogrel, 75 $\mathrm{mg}$ /day) orally 2 days before and continuing after the procedure.

On July 25, 2007, CAS was performed. A guiding catheter $(6 \mathrm{~F}$, Shuttle-SL guide sheath) was positioned in the left common carotid artery. A filter-type catheter (5- $\mathrm{mm}$ in diameter and $300 \mathrm{~cm}$ in length, MintCatch; IR Medical Koubou, Koriyama, Fukushima, Japan) (Fig $2 B$ ) was navigated through the carotid bifurcation stenosis and positioned in the distal ICA as a distal protection device. A low-profile 4.0-mm-diameter balloon catheter (Gateway; Boston Scientific) was advanced to the carotid stenosis over the filter wire and dilated up to $6 \mathrm{~atm}$. The balloon catheter was replaced by a self-expandable stent $(8 \times 40 \mathrm{~mm}$ Precise; Cordis), which was deployed over the residual stenosis. No complications occurred during and after the procedures. Neither transient ischaemic attacks nor strokes occurred during the follow-up. On October 30, 2007, left carotid angiography showed complete obliteration of the aneurysm and sufficient dilation of the carotid artery after CAS (Fig $3 A,-B$ ).

\section{Discussion}

Cerebral aneurysms are incidentally found at autopsy in approximately $5 \%$ of adults. ${ }^{5}$ As for the treatment of unruptured cerebral aneurysms, neurosurgical clipping has been indicated for patients with a life expectancy of more than 5 years, younger than 70 years of age, and harboring an aneurysm of $\geq 5 \mathrm{~mm}^{6-9}$ It is reported ${ }^{10}$ that endovascular coiling can allow more patients with ruptured intracranial aneurysms to become independent at 1 year than neurosurgical clipping. van 



Fig 1. Left common carotid angiography (anteroposterior projection) shows a high-grade carotid bifurcation stenosis $(A)$ and a coincidental unruptured intracranial aneurysm $(6 \times 4$ mm) (B).


Fig 2. A, Left common carotid angiography (anteroposterior projection) after positioning the guide-catheter (6F Shuttle-SL guide sheath) demonstrates the support-wire (0.014-inch Right Away Super Hard) inserting into the left ECA and a high-grade carotid bifurcation stenosis. B, MintCatch filter-type catheter with a 5-mm diameter.

Rooij et al $^{11}$ also reported that coiling should be the first treatment option in incidental aneurysms. Results of cooperative studies have clearly demonstrated the benefit of surgery for patients with severe symptomatic carotid stenosis. ${ }^{12,13}$ Among patients with severe carotid artery stenosis and coexisting high-risk conditions, CAS with the use of an emboli-protection device is not inferior to carotid endarterectomy (CEA). ${ }^{14,15}$

Cerebral unruptured aneurysms ipsilateral to a carotid lesion producing symptoms are often found incidentally in the most common situation. Of the 2885 patients participating in the North American Symptomatic Carotid Endarterectomy Trial (NASCET), 51 (1.7\%) had ipsilateral unruptured intracranial aneurysms. ${ }^{2}$ Patients with a symptomatic cervical carotid bifurcation stenosis coupled with a coexisting cerebral aneurysm in the carotid distal segment present a therapeutic dilemma. ${ }^{1}$ Relief of the carotid stenosis, leading to a sudden rise in perfusion pressure, might increase the potential risk of aneurysm rupture. ${ }^{1-4}$ Conversely, repair of the aneurysm with the patient under general anesthesia may increase the risk of strokes because of decreased CBF imposed by the carotid stenosis. $^{1,2}$

By analyzing the results of NASCET, ${ }^{13}$ a previous study reported $^{2}$ that 25 patients with ipsilateral unruptured aneurysms underwent CEA on the side of their symptomatic artery without having them repaired and that subarachnoid hemorrhage occurred in 1 (4\%) of the 25 patients 6 days after CEA. The patient died 4 days after subarachnoid hemorrhage. This result suggests that a $4 \%$ aneurysm-rupture rate is high, and repair of unruptured aneurysms before CEA is a rational approach. In patients with a severe carotid stenosis, however, neurosurgical clipping of an unruptured intracranial aneurysm with the patient under general anesthesia may increase the perioperative risk of ischemic stroke. Even though CAS before neurosurgical clipping is preferred, a sudden rise in perfusion pressure and subsequently required high-dose antiplatelets may increase the risk of aneurysm rupture. Although aneurysm coiling first is a rational approach, particularly in case of procedures performed under local anesthesia, there are technical problems concerning how to embolize aneurysms 

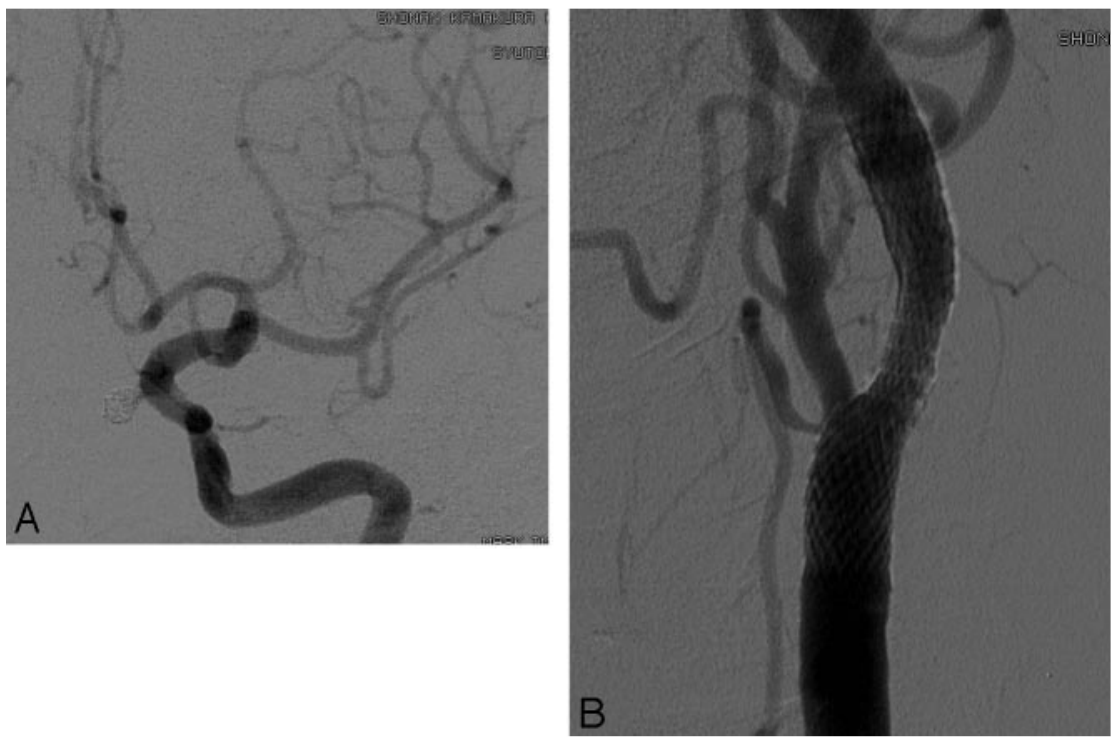

Fig 3. Left common carotid angiography 3 months after CAS (anteroposterior projection) shows complete obliteration of the embolized aneurysm (A) and no restenosis of the carotid bifurcation $(B)$.

through the cervical carotid bifurcation stenosis. Unless the ipsilateral intracranial arteries are opacified well after placing a microcatheter through the severe carotid stenosis ( $\geq 95 \%)$, dilation of the carotid stenosis before coiling is required. If the intracranial arteries are opacified well even after placing a microcatheter through the severe carotid stenosis, the ipsilateral aneurysm can be treated first.

Treatment strategy must be planned regarding safety, which is the highest priority, particularly in an elective procedure in a patient with a carotid stenosis coupled with an ipsilateral unruptured aneurysm. To reduce the risk of aneurysm rupture following carotid dilation, complete repair of aneurysms before carotid dilation is expected. Aneurysms do not heal immediately after coiling; a few months are required for complete repair. After angiographically confirming that the aneurysm is still obliterated at least 1 month after coiling, carotid stent placement is performed.

In case of a symptomatic cervical carotid bifurcation stenosis coupled with a coexisting cerebral aneurysm in the carotid distal segment, staged endovascular treatment is a rational option, and a support-wire technique through a guide catheter with a large internal diameter is very useful for stability of the procedures during aneurysm coiling, unless peripheral blood flow of the distal ICA is disturbed during the procedures.

\section{Acknowledgments}

We acknowledge the secretarial assistance of Nozomi Chiba and the specialized assistance of nurses and radiologic technicians in our neuroendovascular catheterization department.

\section{References}

1. Ladowski JS, Webster MW, Yonas HO, et al. Carotid endarterectomy in patients with asymptomatic intracranial aneurysm. Ann Surg 1984;200:70-73

2. Kappelle LJ, Eliasziw M, Fox AJ, et al. Small, unruptured internal aneurysms and management of symptomatic carotid artery stenosis. Neurology 2000;55:307-09

3. Adams HP Jr. Carotid stenosis and coexisting ipsilateral intracrania aneurysm: a problem in management. Arch Neurol 1977;34:515-16

4. Pappada G, Fiori L, Marina R, et al. Management of symptomatic carotid stenoses with coincidental intracranial aneurysms. Acta Neurochir (Wien) 1996;138:1386-90

5. McCormick WF, Schochet SS. Atlas of Cerebrovascular Diseases. Philadelphia: WB Saunders; 1976:49

6. Chang HS, Kirino T. Quantification of operative benefit for unruptured cerebral aneurysms: a theoretical approach. J Neurosurg 1995;83:413-20

7. Kassell NF, Torner JC. Size of intracranial aneurysm. Neurosurgery 1983;12: 291-97

8. King JT, Berlin JA, Flamm ES. Mortality and morbidity from elective surgery for symptomatic, unruptured cerebral aneurysm. J Neurosurg 1994;81:837-42

9. King JT, Glick HA, Mason TJ, et al. Elective surgery for asymptomatic, unruptured intracranial aneurysms: a cost-effectiveness analysis. J Neurosurg 1995;83:403-12

10. Molyneux A, Kerr RS, Yu L, et al. International subarachnoid aneurysm trial (ISAT) of neurosurgical clipping versus endovascular coiling in 2143 patients with ruptured intracranial aneurysm: a randomised compaction of effects on survival, dependency, seizure, rebleeding, subgroup, and aneurysm occlusion. Lancet 2005;366:809-17

11. van Rooij WJ, Gast A, Sluzewski M, et al. Coiling of truly incidental intracranial aneurysms. AJNR Am J Neuroradiol 2006;27:293-96

12. MRC European Carotid Surgery Trial: interim results for symptomatic patients with severe (70-99\%) or with mild (0-29\%) carotid stenosis-European Carotid Surgery Trialists' Collaborative Group. Lancet 1991;337:1235-43

13. Beneficial effect of carotid endarterectomy in symptomatic patients with high-grade carotid stenosis: North American Symptomatic Carotid Endarterectomy Trial Collaborators. N Engl J Med 1991;325:445-53

14. Theiss W, Hermanek P, Mathias K, et al. Pro-CAS: prospective registry of carotid angioplasty and stenting. Stroke 2004;35:2134-39

15. Yadav JS, Wholey MH, Kuntz RE, et al. Protected carotid-artery stenting versus endarterectomy in high-risk patients. N Engl J Med 2004;351:1493-501 